\title{
Tissue Engineering and Regenerative Science in Pediatrics
}

\author{
JAMES C. Y. DUNN
}

Departments of Bioengineering and Surgery, University of California, Los Angeles, California 90095

$\mathrm{T}$ ssue engineering is a discipline that focuses on the regeneration of living tissues from a combination of cells, biomaterials, and signaling cues (1). The nature of this regenerative science is multidisciplinary, drawing knowledge from cellular and developmental biology, material science and engineering, drug delivery, and wound healing. There has been a tremendous growth in the number of scientific publications that relates to tissue engineering. A simple search in the PubMed database using the term "tissue engineering" shows that the number of publication has grown from less than one per year in the 1980s to 2087 in 2007. This tremendous growth is partly due to the increased interests in stem cells and their therapeutic potential, leading to a new era in regenerative science. Tissue engineering technology has been applied to many organ systems over the last two decades. Several tissue-engineered products are either in clinical use or in clinical trials. For example, skin substitutes made from human cells and collagen matrices have been used to treat patients with burn injury (2). Urinary bladder replacements made from autologous cells and biodegradable polymers to augment bladders of patients with myelomeningocele are in phase 2 clinical trials (3). These tissue-engineering based treatments bring new options and hope to a wide range of patients with tissue deficits.

The potential applications of tissue engineering to the pediatric population are diverse. Congenital anomalies that require surgical reconstruction of the tissue deficits occur in every organ system. For example, children with a cleft palate may require bone grafts for reconstruction, and bone regeneration using stem cells, biomaterials, and molecular signals can avoid the donor site morbidity $(4,5)$. In the cardiovascular system, children with congenital heart disease may require shunts, patches, and valves for their repair, and the use of cells and biomaterials to produce cardiovascular tissue substitutes are being actively pursued $(6,7)$. In the genitourinary systems, children with hypospadias, dysfunctional bladders, and dysplastic kidneys will often require multiple procedures for urologic reconstruction. Tissue deficits in these settings are being addressed with emerging tissue-engineered products $(8-10)$. The application of tissue engineering is well suited to the pediatric population because children heal better and adapt faster than adults. It is also more

Received March 3, 2008; accepted March 6, 2008.

Correspondence: James C. Y. Dunn, M.D., Ph.D., Department of Bioengineering and Surgery, Mail Code 709818, 10833 Le Conte Avenue, Los Angeles, CA 90095; e-mail: jdunn@mednet.ucla.edu

Supported by National Institutes of Health, Fubon Foundation. challenging because the tissue-engineered organ needs to be designed to allow for the child's growth and development.

The cells used for tissue engineering is often based on stem cells or progenitor cells that can give rise to the cell types in the tissue of interest (11). Cell-based therapies utilizing stem cells may be sufficient to regenerate the tissue of interest. For example, the transplantation of hematopoietic stem cells can reconstitute the hematopoietic system after total body irradiation. The excitement over stem cells' potentials has increased the public's awareness of regenerative science. There are many types of stem cells derived from a variety of sources (12). Pluripotent stem cells such as the mesenchymal stem cells may be used for many tissues, while tissue-specific stem cells such as hepatic stem cells are more restricted. Although pluripotent stem cells are more versatile, their growth and differentiation potential needs to be controlled to avoid possible tumor formation. Tissue-specific stem cells, on the other hand, are predetermined to become the cell type of interest but are more difficult to identify and to expand in vitro. Another important cell source consideration is whether to use an autologous source such as the patient's own bone marrow or an allogeneic source such as the human embryonic stem cell. Autologous cells are ideal when they are available, but they usually require an invasive procedure for their procurement. The allogeneic cells are more easily obtained but will likely require long-term immunosuppression. The recent reports on induced pluripotent cells from an autologous source may provide an alternative to human embryonic stem cells (13).

Biomaterials constitute the second major component of tissue engineering (14). It is well known that cells do not function normally when they are taken out of their usual environment. The creation of the normal cellular niche represents a significant challenge in tissue engineering. Most cell types need to be supported with a substrate to provide the surface area for attachment and the spatial volume for growth. The biomaterial may be derived from synthetic or natural sources. Synthetic materials such as polyglycolic acid polymers have the advantage of being more readily available and more easily customized; however, they tend to exert little biologic effect (15). As an example, the bladder substitute Neo-Bladder employs autologous smooth muscle cells and urothelial cells seeded on a polymer scaffold made from polyglycolic acid to form a reservoir to augment bladder capacity (3). The smooth muscle cells synthesized their own extracellular matrix, and the urothelial cells spontaneous organized into an epithelium. Natural materials such as collagens have more biologic activity but are more difficult to obtain and are less amenable to modifications (16). In the skin substitute Apligraf, allogeneic 
fibroblasts, and keratinocytes seeded on a collagenous matrix accelerated the healing of skin wounds (17). The collagen served as a scaffold for the seeded cells, which were eventually replaced with recipient's skin cells.

Signaling cues represent the third major component of tissue engineering (18). In general, the necessary signals for regeneration are not well defined for most tissues. In some cases, cells seeded in the materials are preprogrammed for regeneration so that no additional signals are needed. When a bioactive material is used as the scaffold, the biomaterial may provide the necessary cues to the seeded cells to guide regeneration. External forces may also be important in influencing cell behavior, especially for cells that normally respond to shear and stress, such as endothelial cells in blood vessels and chondrocytes in cartilage (19). When the dominant signaling cue is a single molecule such as the basic fibroblast growth factor, the delivery of such proteins will enhance tissue regeneration (20). More often, multiple signals are needed at different times, and the controlled delivery of multiple factors to match the needs of the tissue is a challenging task. Another source of signals comes from the delivered cells that can secrete cytokines to facilitate the regeneration. For example, bone marrow stem cells have been delivered to the ischemic myocardium to regenerate cardiac myocytes (21). Although clinical trials have shown improved cardiac function postcellular transplantation, few of the delivered cells actually became cardiac myocytes. Instead, it is believed that the delivered stem cells released factors that recruited collateral vessels to the ischemic tissue (22).

Ultimately, the newly formed tissue needs to be integrated with that of the recipient through the process of wound healing. Depending on the tissue type, the integration may need to occur on multiple levels. For example, neurons need to form the synaptic connections with other neurons and cardiac myocytes need to coordinate their contraction with the rest of the myocardium. One important aspect of the integration is the vascularity of the newly formed tissue. Since nearly all tissues require a blood supply to bring oxygen and nutrients to the cells, the circulatory system needs to be connected with the regenerating tissue. Conceptually, there are two ways to accomplish the circulatory integration. The first way is to implant the cell-seeded biomaterial and to wait for the tissue regeneration to occur in vivo. The angiogenic response from the recipient will provide the necessary blood supply to the tissue during the process of wound healing. This is a commonly used strategy in current tissueengineering efforts; however, the disadvantage lies in the relatively long time needed for the capillaries to penetrate the biomaterials, especially when the dimension exceeds a millimeter. Cells located beyond the diffusion limit will therefore experience significant hypoxia and perish (23). The second way overcomes this limitation by engineering a preformed vascular system so that the tissues can be perfused immediately upon implantation. Such strategies can provide ready oxygen and nutrients to the implanted cells (24).

Tissue engineering is going through a phase of exciting technological development, but the complexity of the regenerative science will require a significant period of further research. The use of stem cells, biomate- rials, and signaling cues for tissue engineering represents a novel therapy that will need to be scrutinized in clinical trials. The expense associated with such research and testing will require a sizeable market to attract the industry to bring these products to commercialization. A substantial commitment from both the private sector and nonprofit organizations will be needed to fund these efforts. Further understanding of the regenerative science will bring about revolutionary treatments to children in need.

\section{REFERENCES}

1. Langer R, Vacanti JP 1993 Tissue engineering. Science 260:920-926

2. Pham C, Greenwood J, Cleland H, Woodruff P, Maddern G 2007 Bioengineered skin substitutes for the management of burns: a systematic review. Burns 33:946-957

3. Atala A, Bauer SB, Soker S, Yoo JJ, Retik AB 2006 Tissue-engineered autologous bladders for patients needing cystoplasty. Lancet 367:1241-1246

4. Panetta NJ, Gupta DM, Slater BJ, Kwan MD, Liu KJ, Longaker MT 2008 Tissue engineering in cleft palate and other congenital malformations. Pediatr Res 63:545551

5. Zuk PA 2008 Craniofacial tissue engineering and the adult stem cell. Pediatr Res 63:478-486

6. Levi DS, Kusnezov N, Carman GP 2008 Applications for "smart materials" in pediatric cardiovascular devices. Pediatr Res 63:552-558

7. Mirensky T, Breuer CK 2008 The development of tissue engineered grafts for reconstructive cardiothoracic surgical applications. Pediatr Res 63:559-568

8. Atala A 2008 Bioengineered tissues for urogenital repair in children. Pediatr Res 63:569-575

9. Perin L, Giuliani S, Sedrakyan S, Da Sacco S, Koh C, De Filippo RE 2008 Stem cell and regenerative science applications in the development of bioengineered renal tissue. Pediatr Res 63:467-471

10. Yamzon J, Kokorowski P, Koh CJ 2008 Stem cells and tissue engineering applications of the genitourinary tract. Pediatr Res 63:472-477

11. Polak J, Mantalaris S 2008 Stem cell bioprocessing - novel biomaterials that activate cells and genes for regeneration and repair of tissue. Pediatr Res 63:461-466

12. Battey JF Jr, Cole LK 2006 A stem cell primer. Pediatr Res 59:1R-3R

13. Takahashi K, Tanabe K, Ohnuki M, Narita M, Ichisaka T, Tomoda K, Yamanaka S 2007 Induction of pluripotent stem cells from adult human fibroblasts by defined factors. Cell 131:861-872

14. Patel M, Fisher JP 2008 Biomaterial scaffolds in pediatric tissue engineering. Pediatr Res 63:497-501

15. Kohane D, Langer R 2008 Polymeric biomaterials in tissue engineering. Pediatr Res 63:487-491

16. Cen L, Liu W, Cui L, Zhang W, Cao YL 2008 Collagen tissue engineering: development of novel biomaterials and applications. Pediatr Res 63:492-496

17. Eaglstein WH, Falanga V 1997 Tissue engineering and the development of Apligraf, a human skin equivalent. Clin Ther 19:894-905

18. Dudas M, Wysocki A, Gelpi B, Tuan TL 2008 Cellular and molecular basis of wound repair and tissue regeneration. Pediatr Res 63:502-512

19. Kobsa S, Saltzman M 2008 Bioengineering approaches to controlled protein delivery. Pediatr Res 63:513-519

20. Williams GM, Klisch SM, Sah RL 2008 Bioengineering cartilage growth, maturation and form. Pediatr Res 63:527-534

21. Nasseri BA, Kukucka M, Dandel M, Knosalla C, Potapov E, Lehmkuhl HB, Meyer R, Ebell W, Stamm C, Hetzer 2007 Intramyocardial delivery of bone marrow mononuclear cells and mechanical assist device implantation in patients with end-stage cardiomyopathy. Cell Transplant 16:941-949

22. Mishra PK 2008 Bone marrow-derived mesenchymal stem cells for treatment of heart failure: is it all paracrine actions and immunomodulation? J Cardiovasc Med (Hagerstown) 9:122-128

23. Dunn JC, Chan WY, Cristini V, Kim JS, Lowengrub J, Singh S, Wu BM 2006 Analysis of cell growth in three-dimensional scaffolds. Tissue Eng 12:705-716

24. Hoganson DM, Pryor HI II, Vacanti JP 2008 Tissue engineering and organ structure: a vascularized approach to liver and lung. Pediatr Res 63:520-526 